\title{
Perception d'espèces agroforestières et de leurs services écosystémiques par trois groupes ethniques du bassin versant de Boura, zone soudanienne du Burkina Faso
}

Mohamed CISSÉ ${ }^{1}$

Babou André BATIONO ${ }^{2}$

Salifou TRAORÉ ${ }^{1}$

Issaka Joseph Boussım ${ }^{1}$

${ }^{1}$ Université Ouaga I Pr Joseph Ki-Zerbo Laboratoire de biologie et écologie végétales

UFR Sciences de la Vie et de la Terre 03 BP 7021, Ouagadougou 03

Burkina Faso

${ }^{2}$ Institut de l'environnement et de recherches agricoles

Département environnement et forêts (INERA/DEF)

04 BP 8645, Ouagadougou 04

Burkina Faso

\section{Auteur correspondant /}

Corresponding author:

Mohamed Cissé - cisamed35@yahoo.fr

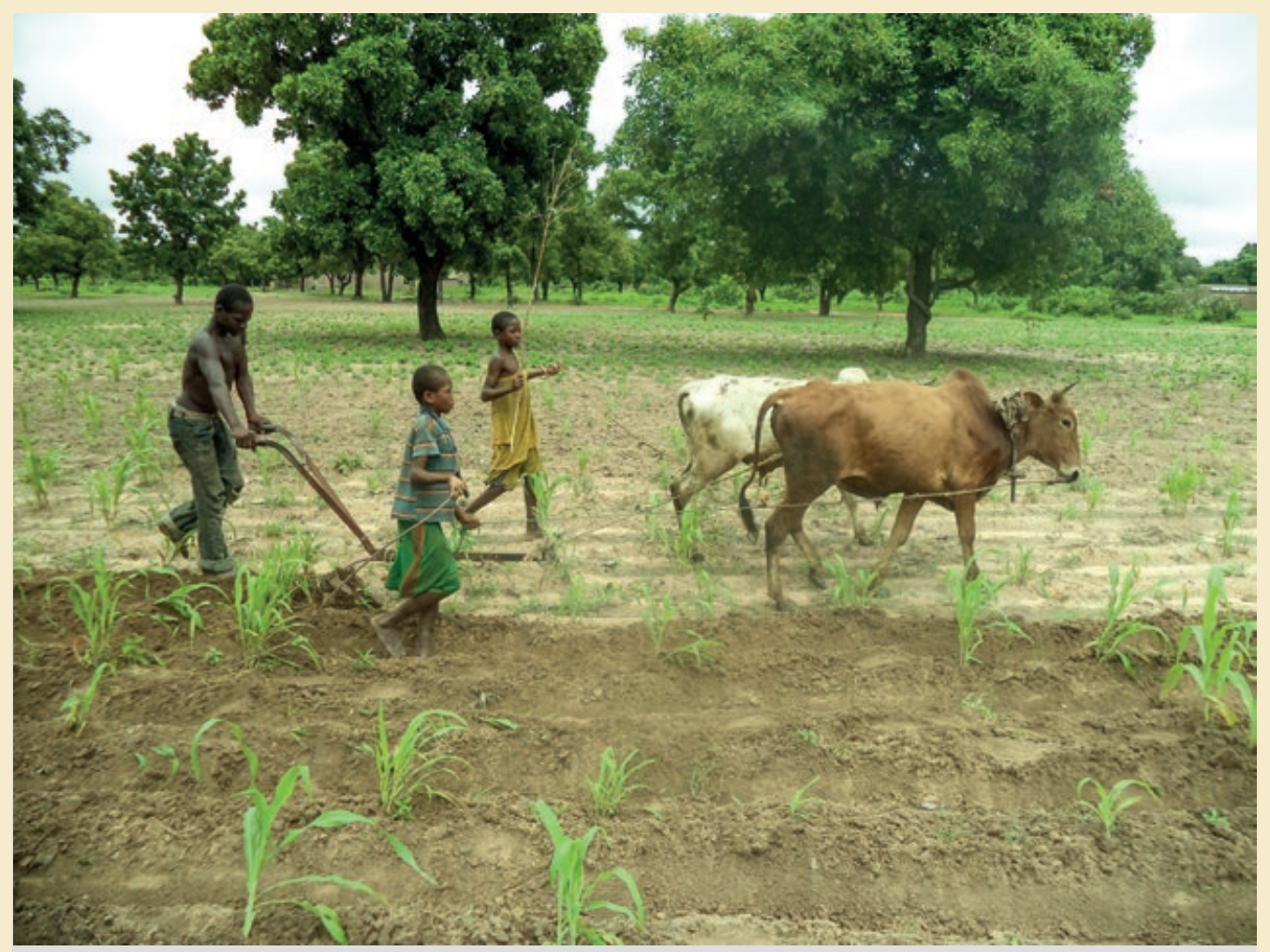

Photo 1.

Billonnage dans un parc agroforestier à Vitellaria paradoxa associé aux cultures de sorgho (Sorghum bicolor) et d'arachide (Arachis hypogaea).

Photo M. Cissé.

Doi : 10.19182/bft2018.338.a31680 - Droit d'auteur (c) 2018, Bois et Forêts des Tropiques @ C Cirad - Date de soumission : 18 février 2018 ; date d'acceptation : 22 juin 2018 ; date de publication : $1^{\text {er }}$ décembre 2018.

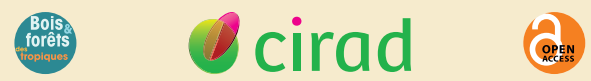

(c) $(1) \Theta$

Licence Creative Commons

Attribution - Pas de Modification 4.0 International.

Attribution-NoDerivatives 4.0 International (CC BY-ND 4.0)
Citer l'article / To cite the article

Cissé M., Bationo B. A., Traoré S., Boussim I. J., 2018. Perception d'espèces agroforestières et de leurs services écosystémiques par trois groupes ethniques du bassin versant de Boura, zone soudanienne du Burkina Faso. Bois et Forêts des Tropiques, n 338 : 29-42. Doi : https://doi. org/10.19182/bft2018.338.a31680 


\section{RÉSUMÉ}

\section{Perception d'espèces agroforestières et de leurs services écosystémiques par trois groupes ethniques du bassin versant de Boura, zone soudanienne du Burkina Faso}

En zone tropicale, les services écosystémiques associés aux espèces agroforestières sont peu connus. Une enquête ethnobotanique a été conduite dans le bassin versant de Boura pour appréhender la perception des espèces agroforestières et leurs services écosystémiques. Des interviews semi-structurées et des observations directes ont été réalisées auprès de 214 chefs d'exploitation (CE) appartenant aux groupes ethniques Sissala, Dagara et Mossi. Les caractéristiques socio-économiques des $\mathrm{CE}$, les espèces ligneuses conservées dans les champs et leurs services écosystémiques ont été recensés. L'importance des espèces a été déterminée selon la méthode de l'indice culturel d'importance $(\mathrm{Cl})$. Des tests de comparaison des réponses et des modèles linéaires généralisés ont été réalisés à l'aide du logiciel R.3.3.2. Au total, 64 espèces ligneuses appartenant à 59 genres et 30 familles ont été recensées. Ces espèces fournissent aux communautés 17 services écosystémiques répartis en quatre catégories. Vitellaria paradoxa $\left(\mathrm{IC}_{\mathrm{T}}=10,45\right)$ et Parkia biglobosa $\left(I C_{T}=7,80\right)$ sont deux espèces agroforestières clés pourvoyeuses de services écosystémiques à l'ensemble des communautés. L'ordination non métrique de la matrice d'occurrence des espèces agroforestières indique qu'en dépit des similitudes de connaissances ethnobotaniques les groupes ethniques Dagara et Sissala manifestent différentes préférences dans la conservation d'essences ligneuses spécifiques dans les champs. Le groupe ethnique, le genre, la taille du ménage, le niveau d'éducation et l'expérience du chef d'exploitation sont les principaux facteurs déterminant la perception et la conservation des espèces agroforestières. La sélection d'essences végétales pour les interventions agroforestières doit tenir compte des facteurs socio-économiques déterminant les préférences des communautés.

Mots-clés : agroforesterie, indice culturel d'importance, services écosystémiques, bassin versant, biodiversité, Burkina Faso.

\section{ABSTRACT}

\section{Perceptions of agroforestry species and their ecosystem services in three ethnic groups in the Boura catchment basin in the Sudanian zone of Burkina Faso}

In tropical zones, there is little knowledge about the ecosystem services associated with agroforestry species. An ethnobotanical survey was undertaken in the Boura catchment basin to identify perceptions of agroforestry species and the ecosystem services they provide. Semi-structured interviews and direct observations were conducted among 214 farmers of the Sissala, Dagara and Mossi ethnic groups. The study identified the socio-economic characteristics of the farmers, the woody species they keep in their fields and the ecosystem services that these provide. The importance of the species was determined by applying the Cultural Importance Index (CI). Comparative tests of the responses and generalised linear models were produced using R.3.3.2 software. Altogether, 64 woody species from 59 genera and 30 families were identified. These species provide the communities with 17 ecosystem services that fall into 4 categories. Vitellaria paradoxa $\left(\mathrm{IC}_{\mathrm{T}}=10.45\right)$ and Parkia biglobosa $\left(\mathrm{IC}_{\mathrm{T}}=7.80\right)$ are both key agroforestry species providing ecosystem services to all the communities. Non-metric multidimensional scaling of the occurrence matrix for agroforestry species suggests that despite their similar ethnobotanical knowledge, the Dagara and Sissala ethnic groups have different preferences for the specific woody species they keep in their fields. The main factors determining perceptions and conservation of agroforestry species are ethnic group, gender, household size, level of education and the experience of the head of the farm. The selection of plant species for agroforestry projects must take account of the socio-economic factors that determine the preferences of communities.

\section{Keywords: agroforestry, Cultural}

Importance Index, ecosystem services, catchment basin, biodiversity, Burkina Faso.

\author{
Percepción de especies agroforestales y \\ de sus servicios ecosistémicos por tres \\ grupos étnicos de la cuenca vertiente de \\ Boura, zona del sur de Burkina Faso
}

En las zonas tropicales, los servicios ecosistémicos asociados a las especies agroforestales son poco conocidos. En la cuenca vertiente de Boura se llevó a cabo una investigación etnobotánica para comprender la percepción de las especies agroforestales y sus servicios ecosistémicos. Se realizaron entrevistas semiestructuradas y observaciones directas a unos 214 jefes de explotación (JE) pertenecientes a los grupos étnicos Sissala, Dagara y Mossi. Se recogieron las características socioeconómicas de los JE, las especies leñosas conservadas en los campos y sus servicios ecosistémicos. La importancia de las especies fue determinada según el método del índice cultural de importancia $(\mathrm{Cl})$. Se realizaron pruebas de comparación de respuestas y modelos lineales generalizados con la ayuda del programa informático R.3.3.2. En total, se censaron 64 especies leñosas pertenecientes a 59 géneros y 30 familias. Estas especies proporcionan a las comunidades 17 servicios ecosistémicos distribuidos en cuatro categorías. Vitellaria paradoxa $\left(I C_{T}=10,45\right)$ y Parkia biglobosa $\left(I C_{T}=7,80\right)$ son dos especies agroforestales clave que proporcionan servicios ecosistémicos al conjunto de las comunidades. La ordenación no métrica de la matriz de ocurrencias de las especies agroforestales indica que, a pesar de las similitudes en conocimientos etnobotánicos, los grupos étnicos Dagara y Sissala manifiestan distintas preferencias en la conservación de especies leñosas específicas en los campos. El grupo étnico, el género, el número de miembros del hogar, el nivel educativo y la experiencia del jefe de explotación son los principales factores que determinan la percepción y la conservación de las especies agroforestales. La selección de especies vegetales para las intervenciones agroforestales debe tener en cuenta los factores socioeconómicos que determinan las preferencias de las comunidades.

Palabras clave: agroforestería, índice cultural de importancia, servicios ecosistémicos, cuenca vertiente, biodiversidad, Burkina Faso. 


\section{Introduction}

Dans les zones arides et semi-arides de l'Afrique de l'Ouest, les parcs agroforestiers (photo 1) sont un type courant d'utilisation des terres (Raison, 1988 ; Bonkoungou et al., 1994 ; Bationo, 2010) dans lesquels des espèces ligneuses sont délibérément maintenues dans les exploitations agricoles, en association avec les cultures, pour de multiples usages (Orwa et al., 2009). Ces arbres procurent aux communautés rurales des services écosystémiques, telle la production de biens alimentaires et médicinaux, de fourrage et de bois (Yaméogo et al., 2005 ; Belem et al., 2008 ; Ngom et al., 2014). Ils assurent également une contribution à la spiritualité (Bationo et al., 2010), et servent à fertiliser les sols (Zomboudre et al., 2005) et à atténuer les effets du changement climatique (Bayala et al., 2014).

Cependant, dans un contexte de pression démographique, de changement climatique et de fortes mutations socio-économiques caractérisées par la mécanisation de l'agriculture, les parcs agroforestiers sont en dégradation continue (Boffa, 2000). La dégradation des ressources agroforestières constitue par conséquent une sérieuse menace pour la résilience des agrosystèmes et des populations rurales qui en dépendent.

Il apparaît impératif d'assurer une documentation inclusive des connaissances ethnobotaniques, nécessaire à la construction de systèmes innovants et durables de régénération et de gestion participative des espèces agroforestières. Des facteurs socio-économiques tels que l'âge, le sexe et les migrations influencent la connaissance (Deb et al., 2009 ;
Brandt et al., 2013 ; Hounsode et al., 2016) et la domestication des espèces ligneuses. La connaissance des services écosystémiques liés aux espèces ligneuses et la compréhension des perceptions des différentes communautés et générations constituent par conséquent une étape indispensable à la sélection d'essences forestières pour la mise au point de technologies agroforestières diversifiées et performantes.

L'objectif général de cette étude était d'appréhender la perception locale des espèces agroforestières et de leurs services écosystémiques dans le bassin versant de Boura. De façon spécifique, elle visait à identifier les espèces agroforestières dans le bassin versant de Boura, à caractériser et comparer les services écosystémiques associés aux espèces agroforestières chez trois groupes ethniques qui cohabitent dans la zone, enfin à déterminer les facteurs socio-économiques et les contraintes qui influencent l'intégration des espèces ligneuses dans les exploitations agricoles.

\section{Méthodologie}

\section{Site d'étude}

Le site d'étude est le bassin versant de Boura (figure 1) situé dans les provinces de la Sissili et du loba, au sud du Burkina Faso, et limité par la République du Ghana (PNGT 2 , 2007). Le bassin versant de Boura, d'une superficie de 40270 ha, comprend une retenue d'eau de 163 ha et des périmètres agricoles aménagés de 113 ha. Le climat est soudanien, avec une pluviométrie moyenne annuelle de $908 \pm 90 \mathrm{~mm}$ et une température moyenne annuelle de $28,4 \pm 2,4^{\circ} \mathrm{C}$ entre 1986 et 2015 .

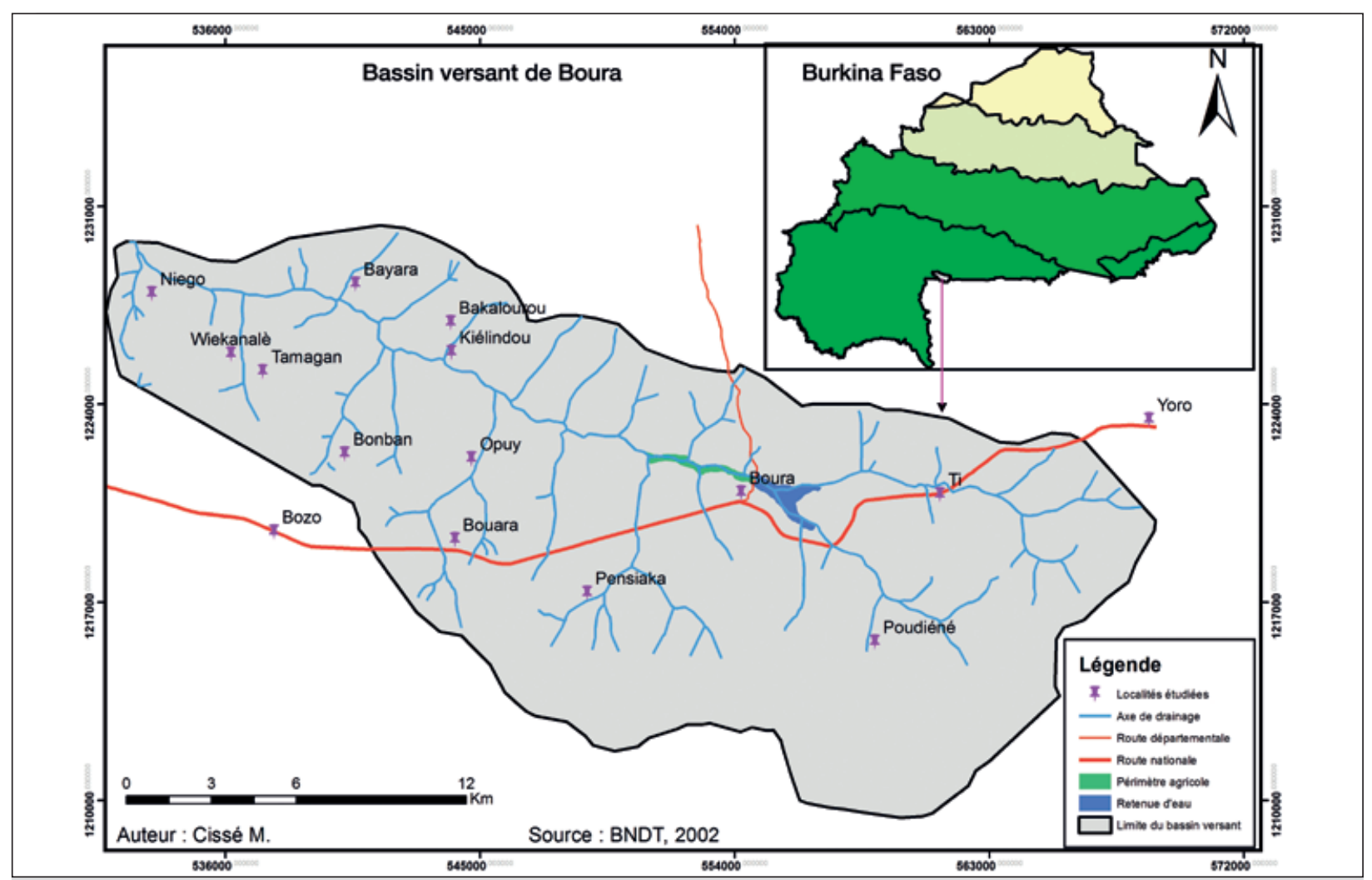

Figure 1.

Carte de localisation de la zone d'étude. 
Bois et Forêts des Tropiques - ISSN: L-0006-579X

Volume 338 - $4^{\text {rd }}$ quarter - December 2018 - p. 29-42

32

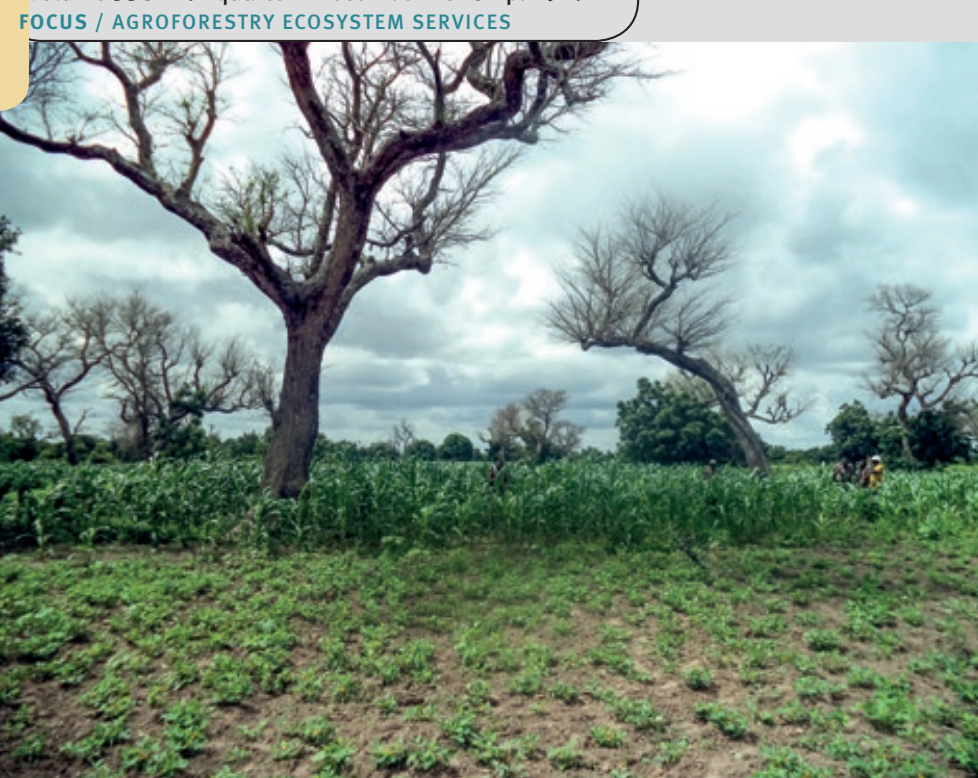

Photo 2.

Parc agroforestier à Faidherbia albida.

Photo M. Cissé.

Le bassin versant de Boura est situé dans le secteur phytogéographique sud-soudanien, avec une végétation essentiellement composée de savanes arborées et arbustives et de galeries forestières (Fontès et Guinko, 1995). Les agrosystèmes sont caractérisés par des parcs agroforestiers (photo 2) avec une présence remarquable d'espèces comme Vitellaria paradoxa, Parkia biglobosa et Faidherbia albida. La végétation d'ensemble est riche en flore humide soudano-guinéenne (Fontès et Guinko, 1995). Isoberlinia doka, Anogeissus leiocarpa, Burkea africana, V. paradoxa, P. biglobosa, Piliostigma thonningii, Terminalia avicennioides sont les espèces ligneuses les plus fréquentes. Le tapis graminéen est souvent dominé par des espèces pérennes telles que Andropogon gayanus, Hyparrhenia glabriuscula, Vetiveria nigritana.

Les types de sols dominants sont des sols ferrugineux tropicaux lessivés, des lithosols et des sols hydromorphes. Le principal cours d'eau du bassin est un affluent semi-permanent du fleuve Mouhoun. Il est alimenté par un réseau de cours d'eau secondaires à régime intermittent, qui ont favorisé la construction d'une retenue d'eau à Boura. La population de la commune de Boura est estimée à 29185 habitants (INSD, 2006), constituée à $55 \%$ d'immigrants, dont 21,6\% de Mossi, 20,2 \% de Dagara et 10,7 \% de Peuls. Les autochtones, Sissala, représentent $47,4 \%$ de la population totale.

L'agriculture extensive de subsistance occupe plus de $90 \%$ de la population active. Les principales productions sont le maïs, le sorgho, le riz, l'igname, le mil et l'arachide. L'élevage est de type extensif et s'organise autour des principales espèces de bovins, caprins et ovins dont le cheptel total atteignait 187730 têtes en 2007 (PNGT 2, 2007). La cueillette traditionnelle porte sur les feuilles, fruits (photo 3 ) et graines ou amandes d'espèces agroforestières comme $P$. biglobosa, V. paradoxa, Detarium microcarpum, Tamarindus indica. Cette activité constitue une source de revenus substantiels, notamment pour les femmes. L'exploitation du bois et du charbon de bois, assurée essentiellement par les hommes, est très importante dans le terroir de Boura.

\section{Échantillonnage et collecte de données}

Des entretiens ont été réalisés avec les populations locales afin d'expliquer la méthode d'étude et obtenir leur participation. Cette étape avait aussi pour but d'identifier de façon participative les services écosystémiques associés aux arbres de champs et de tester le guide d'entretien. Pendant les campagnes agricoles 2015-2016, des enquêtes selon des entretiens semi-structurés (ISS) et des observations directes dans les champs ont été conduites auprès des chefs d'exploitation (CE). Dans la présente étude, le CE désigne la personne qui dirige une exploitation agricole. Les CE ont été choisis comme informateurs (Albuquerque et al., 2014) en raison de leur expérience et connaissance des parcs agroforestiers (Abegg et al., 2006 ; Kalinganire et al., 2005). L'ancienneté du CE est son nombre d'années d'expérience (NAE) dans la gestion d'une exploitation. Au total, 214 CE dont 98 femmes et 116 hommes ont été interviewés dans trois groupes ethniques (tableau I) : les Mossi, les Sissala et les Dagara. Le guide d'entretien était axé sur les caractéristiques socio-économiques du CE, les espèces ligneuses conservées dans les champs, et les services écosystémiques qui leur sont associés. Les critères de sélection des individus d'espèces à conserver et les contraintes de gestion des parcs agroforestiers ont été également recensés. Nous considérons que les services écosystémiques désignent tous les biens et bénéfices environnementaux que procurent les espèces agroforestières. Quatre catégories de services écosystémiques ont été retenues sur la base de la classification du MEA (2005) : les services d'approvisionnement, les services de régulation, les services de support et les services culturels. Au cours des excursions dans les champs, les espèces ligneuses ont été directement identifiées et leur nom en langue locale a été noté. Des échantillons d'herbier ont aussi été collectés et identifiés à l'aide des flores. Les noms des espèces et des familles ont été mis à jour selon la nomenclature adoptée dans le catalogue des plantes vasculaires du Burkina Faso (Thiombiano et al., 2012a). La liste floristique établie a été comparée à celle des espèces menacées et bénéficiant d'une protection particulière au Burkina Faso.

\section{Traitement et analyse de données}

La fréquence de présence ( $\mathrm{Fp}$ ) des espèces ligneuses dans les champs a été calculée pour chaque groupe ethnique selon la formule suivante :

$\mathrm{Fp}=\frac{S}{N} \times 100$

où $S$ représente le nombre de fois qu'une espèce a été recensée dans les champs et $N$ est le nombre total des enquêtés.

Le pourcentage de citation des services dans chaque catégorie a été calculé en multipliant par cent le nombre de services rapportés, puis en divisant par le nombre total de services recensés dans la catégorie.

L'indice culturel d'importance (IC) a été utilisé pour appréhender la variabilité des connaissances liées aux espèces agroforestières, selon la formule de Tardío et Pardo-de-Santayana (2008) réadaptée : 
Tableau I.

Répartition des échantillons des 214 chefs d'exploitation enquêtés.

Catégorie sociale

Vieux hommes (> 50 ans)

Vieilles femmes (> 50 ans)

Jeunes hommes ( $\leq 50$ ans)

Jeunes femmes ( $\leq 50$ ans)

Total par ethnie

$I C=\sum_{S=s 1}^{S_{N u}} \sum_{I=1}^{I=n} \frac{S R s_{i}}{N}$

Avec Nu : nombre total de catégories de services; $S R$ : nombre de services rapportés; $S_{i}$ : nombre de services mentionnés par un informateur $i$; $N$ nombre total d'informateurs.

Ainsi, la valeur limite de l'indice culturel d'importance pour une espèce est le nombre total de services retenus pour l'étude.

Dans un contexte de mutations sociales qui affectent les connaissances associées aux ressources végétales, l'indice culturel d'importance est approprié pour comparer entre groupes ethniques les perceptions liées aux espèces agroforestières (Tardío et Pardo-de-Santayana, 2008 ; Houéhanou et al., 2015).

Le test de corrélation de Pearson a été effectué afin d'évaluer la relation entre la fréquence de présence et l'indice culturel d'importance. Une ordination non métrique (NMS) a été réalisée sur la matrice d'occurrence des espèces pour discriminer les préférences des espèces agroforestières chez les groupes ethniques.

L'effet des facteurs socio-économiques que sont l'ethnie, le niveau d'éducation et le statut résidentiel sur le nombre d'espèces épargnées a été analysé à l'aide du test non paramétrique de Kruskal-Wallis au seuil de $5 \%$. Le modèle linéaire généralisé (GLM) utilisant la distribution de Poisson et la fonction de lien logarithmique a permis de déterminer l'impact des facteurs socioéconomiques sur la perception des espèces agroforestières. Ainsi l'âge, le nombre d'années d'expérience, la taille des ménages et le nombre d'actifs ont été considérés comme des variables explicatives, le nombre d'espèces épargnées étant la variable expliquée. Les données ont été analysées à l'aide du logiciel R.3.3.2.

\section{Résultats}

\section{Caractéristiques socio-économiques des chefs d'exploitation}

L'âge moyen des chefs d'exploitation est de $50,0 \pm 11,5$ ans (tableau II), avec une moyenne de 17,5 $\pm 11,1$ ans d'ancienneté dans la gestion des parcs. Parmi les chefs d'exploitation enquêtés, 52,8\% sont des migrants.

\section{Diversité des espèces ligneuses épargnées dans les champs}

Un total de 64 espèces ligneuses réparties dans 59 genres et 30 familles a été recensé dans les champs (tableau V). Les familles les plus représentées (figure 2) sont les Fabaceae-Caesalpinoidae (14\%) suivies des Fabacae-Mimosoidae (9\%). Selon les CE, les principaux critères de sélection des individus d'espèces ligneuses à maintenir dans les champs sont l'importance socio-économique de l'espèce (100\%), la valeur socioculturelle $(42,5 \%)$ et la rareté des espèces $(42,5 \%)$. $V$. paradoxa $(100 \%)$ et $P$. biglobosa $(80,5 \%)$ sont les plus fréquentes dans les champs, quel que soit le groupe ethnique considéré (figure 3). Les espèces recensées dans les champs représentent $60 \%$ des espèces végétales bénéficiant d'une protection particulière au Burkina Faso.

\section{Espèces agroforestières préférées dans les parcs agroforestiers}

L'ordination non métrique sépare les espèces agroforestières en fonction des groupes ethniques, du sexe et des classes d'âge. Les deux premiers axes de la NMS expliquent $71,3 \%$ de la variance totale. Suivant l'axe 1, les connaissances ethnobotaniques des hommes s'opposent à celles des femmes. L'axe 2 sépare les espèces agroforestières préférées par les vieux de celles des jeunes dans les groupes ethniques. Ainsi, les Dagara conservent préférentiellement Strychnos spinosa et Cordia mixa tandis que les Sissala maintiennent spécifiquement $D$. microcarpum, Lannea
Tableau II.

Caractéristiques socio-économiques des chefs d'exploitation.

\begin{tabular}{l|c|c|c|c|}
$\begin{array}{l}\text { Variables } \\
\text { socio-économiques }\end{array}$ & Âge (ans) & $\begin{array}{c}\text { Ancienneté } \\
\text { (ans) }\end{array}$ & $\begin{array}{c}\text { Taille du } \\
\text { ménage }\end{array}$ & $\begin{array}{c}\text { Fréquence } \\
\text { (\%) }\end{array}$ \\
$\begin{array}{l}\text { Sexe } \\
\text { Féminin }\end{array}$ & $48,59 \pm 10,92$ & $13,72 \pm 7,67$ & $7,81 \pm 3,17$ & 45,80 \\
\hline Masculin & $51,32 \pm 11,97$ & $20,76 \pm 12,47$ & $9.87 \pm 5,00$ & 54,40 \\
\hline Ethnie & $49,82 \pm 11,67$ & $14,80 \pm 11,98$ & $9,40 \pm 5,10$ & 35,04 \\
\hline Sissala & $49,65 \pm 10,77$ & $18,68 \pm 9,51$ & $8,83 \pm 4,91$ & 28,03 \\
\hline Mossi & $50,63 \pm 12,13$ & $19,27 \pm 10,97$ & $8,56 \pm 2,99$ & 36,91 \\
\hline Dagara & & & &
\end{tabular}




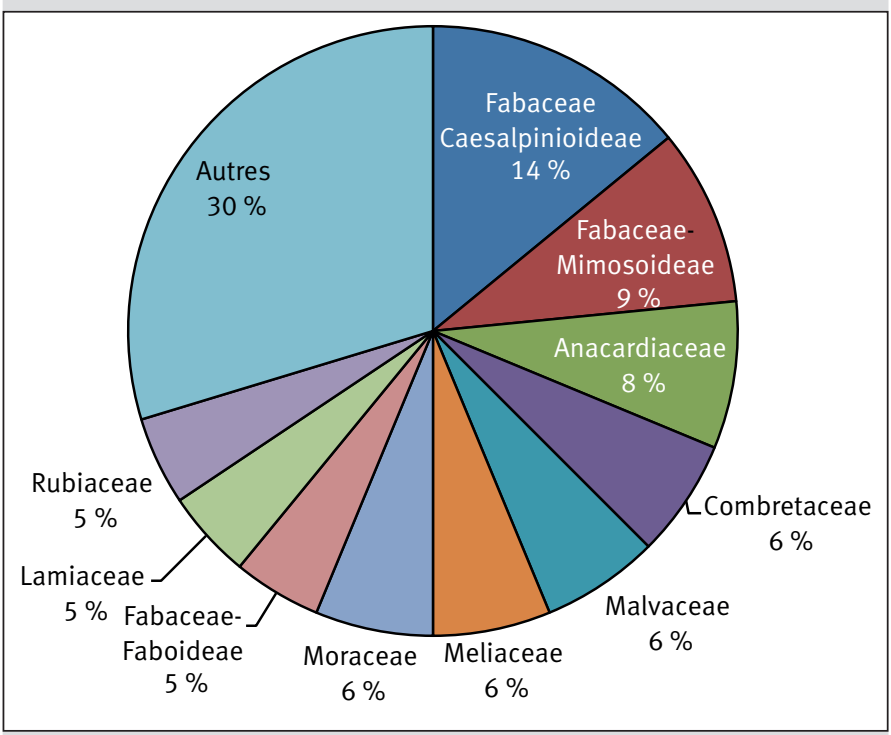

Figure 2.

Spectre de famille des espèces épargnées par les défrichements.

acida, F. albida, Daniellia oliveri, Afzelia africana, Pterocarpus erinaceus, Khaya senegalensis, Bombax costatum et $B$. africana. Dans la zone d'étude, V. paradoxa, $P$. biglobosa, T. indica, Diospyros mespiliformis, Lannea microcarpa, Saba senegalensis, Azadirachta indica, Ficus sycomorus sont des espèces agroforestières intégrées dans les exploitations chez tous les groupes ethniques (figure 4). Aucun groupe d'espèces n'est associé spécifiquement aux Mossi, d'ailleurs la perception des hommes dans ce groupe ethnique se rapproche plus de celle des Sissala.

\section{Services écosystémiques fournis} par les espèces ligneuses dans les parcs agroforestiers

Au cours des rencontres avec les communautés, 17 services écosystémiques associés aux parcs agroforestiers ont été identifiés et regroupés en quatre catégories. Les services de régulation (35\%) sont les plus fréquents, suivis des services d'approvisionnement (31\%) et des services culturels et de support (17\%). Parmi les 17 services écosystémiques recensés, la production d'ombrage (54\%) et la fertilisation des sols (46\%) sont les plus citées (figure 5). Les services les moins cités sont liés au mysticisme (7,3\%), au marquage de territoire $(6 \%)$ et à la production de fourrage $(5,4 \%)$.

\section{Perception des catégories de services écosystémiques selon les groupes ethniques}

L'Indice culturel d'importance montre que les services d'approvisionnement, suivis des services de régulation (tableau V), sont les plus perçus par les communautés de la zone d'étude. Bien que la perception (figure 6) des services écosystémiques soit prépondérante chez les Sissala $(50,0)$ contre 43,8 chez les Mossi et 33,8 chez les Dagara, le test de Kruskal-Wallis indique que l'indice culturel d'importance ne varie pas significativement selon les groupes ethniques $\left(x^{2}=2, d f=2, p\right.$-value $\left.=0,3679\right)$.

\section{Facteurs influençant la connaissance des espèces agroforestières}

Les connaissances liées aux espèces agroforestières dépendent des facteurs socio-économiques (figure 7). Le test de Kruskal-Wallis (tableau III) montre que le nombre d'espèces épargnées est significativement influencé par le niveau d'instruction, l'ethnie et le sexe ( $p$-value $<0,05$ ) du chef d'exploitation (tableau IV). Le nombre moyen d'espèces épargnées est de 3,6 chez les femmes contre 5,6 chez les hommes.

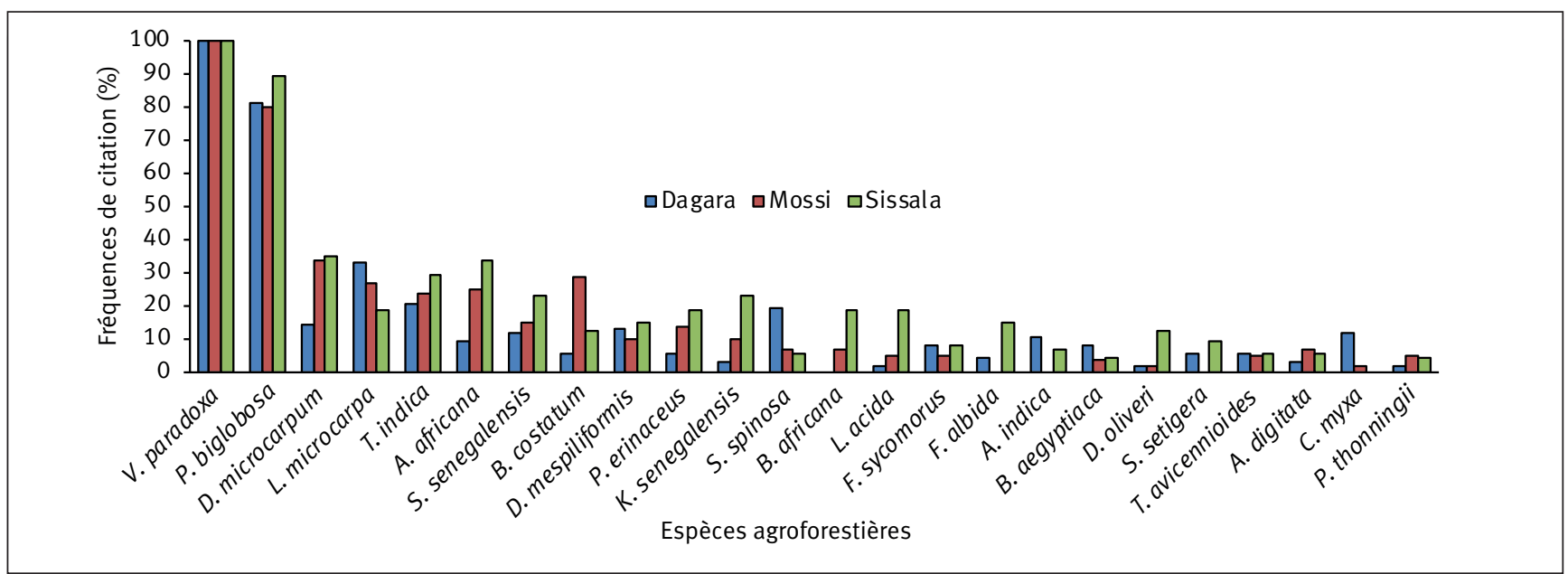

Figure 3.

Fréquences de présence des espèces agroforestières selon les groupes ethniques. 


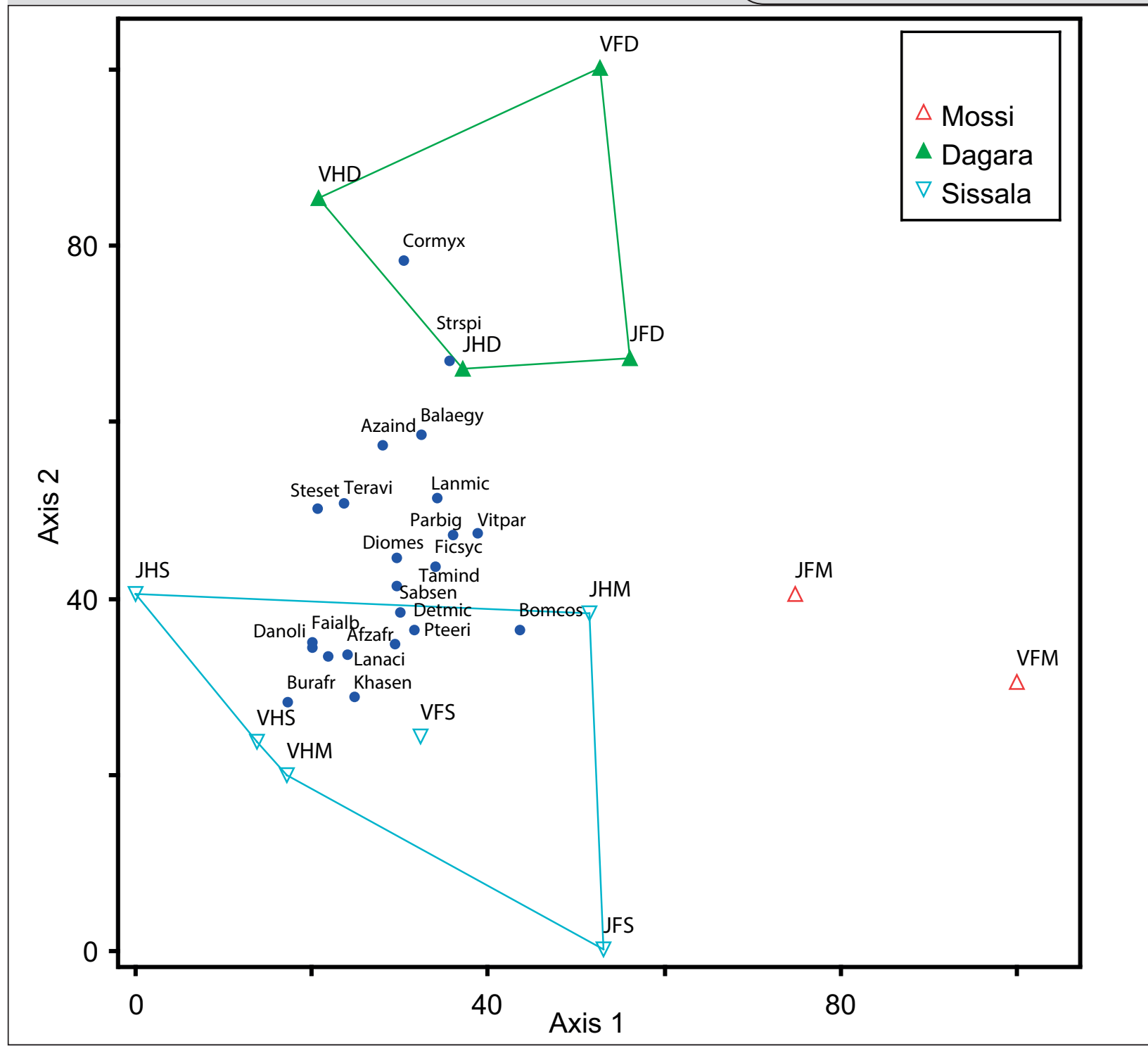

Figure 4.

Ordination non métrique (NMS) des espèces selon les groupes ethniques.

Légende : Vitpar : Vitellaria paradoxa; Parbig : Parkia biglobosa; Detmic : Detarium microcarpum ; Lanmic : Lannea microcarpa ; Tamind : Tamarindus indica ; Afzafr : Afzelia africana ; Ficsyc : Ficus sycomorus ; Sabsen : Saba senegalensis ; Bomcos : Bombax costatum ; Pteeri : Pterocarpus erinaceus; Khasen : Khaya senegalensis ; Teravi : Terminalia avicennioides ; Diomes : Diospyros mespiliformis ; Strspi : Strychnos spinosa ; Burafr : Burkea africana ; Faialb : Faidherbia albida ; Cormyx : Cordia myxa ; Azaind : Azadirachta indica ; Balaegy : Balanites aegyptiaca ; Danoli : Daniellia oliveri ; Lanaci : Lannea acida ; Steset : Sterculia setigera ; JFM : jeune femme Mossi ; JFD : jeune femme Dagara ; JFS : jeune femme Sissala ; JHD : jeune homme Dagara ; JHM : jeune homme Mossi ; JHS : jeune homme Sissala ; VFD : vieille femme Dagara ; VFM : vieille femme Mossi ; VFS : vieille femme Sissala ; VHD : vieil homme Dagara ; VHM : vieil homme Mossi ; VHS : vieil homme Sissala.

Le modèle linéaire généralisé (GLM) indique que l'ancienneté et la taille du ménage ont des effets significativement positifs sur le nombre d'espèces agroforestières (tableau IV).

La corrélation de Pearson entre la fréquence de présence (FP) et l'indice culturel d'importance (IC) est fortement significative $\left(r=0,997, p\right.$-value $\left.<2,2 e^{-16}\right)$. Une classification des espèces agroforestières en fonction des préférences des populations a donc été proposée (tableau $V$ ). Les espèces préférées sont $V$. paradoxa, P. biglobosa, $D$. microcarpum, L. microcarpa, T. indica, A. africana, S. senegalensis, B. costatum, P. erinaceus, $K$. senegalensis, $D$. mespiliformis,
S. spinosa, Burkea africana, Faidherbia albida, A. indica, L. acida, F. sycomorus, T. avicennioides, C. myxa, Balanites aegyptiaca, Sterculia setigera et $D$. oliveri.

\section{Contraintes de gestion des parcs agroforestiers}

Selon $71,5 \%$ des CE, les principales contraintes de gestion des parcs agroforestiers sont les attaques parasitaires $(60 \%)$ et les problèmes avec les agents forestiers, liés à l'application de la réglementation (55\%) sur les arbres entretenus par les paysans dans leurs champs (figure 8). 


\section{Discussion}

Le nombre d'espèces conservées dans les champs varie significativement selon l'ethnie, le sexe, la taille du ménage et l'ancienneté du chef d'exploitation. Il est plus élevé dans les champs des Sissala, autochtones, indiquant que ces derniers détiennent plus de connaissances ethnobotaniques sur les espèces agroforestières que les migrants Mossi et Dagara. Les Sissala ont ainsi des pratiques agricoles qui préservent mieux les plantes ligneuses (Bationo et al., 2004) et constituent un sous-groupe des Gourounsi dont les connaissances ethnobotaniques de la végétation locale ont été déjà mises en évidence (Kristensen et Lykke, 2003).
Dans la zone d'étude, 64 espèces ligneuses ont été inventoriées dans les champs. Dao (2013) avait relevé 20 espèces maintenues dans les champs dans la zone de Fada et Dédougou ; Yaméogo et al. (2005) en avaient observé 44 dans le terroir de Vipalgo, en zone nord-soudanienne du Burkina Faso. Ces différences sont sans doute liées à notre échantillonnage qui a pris en compte trois groupes ethniques, à la zone agroécologique retenue, et aux connaissances et pratiques locales qui favorisent la conservation des ligneux. Ce terme de conservation est d'autant plus approprié que $40 \%$ des espèces ligneuses conservées dans les champs sont menacées au Burkina Faso (Ganaba, 2010), et que les espèces présentes recouvrent $60 \%$ des espèces végétales bénéficiant d'une protection intégrale au Burkina Faso (MECV, 2004). L'espace champêtre constitue donc un refuge pour les espèces menacées.

L'ordination non métrique indique que les espèces ligneuses sont différemment préférées par les groupes ethniques qui ne maintiennent que certaines espèces dans leurs champs. C'est le cas de S. spinosa et C. mixa qui sont préservées par les Dagara. Il existe beaucoup de similitudes entre les connaissances des Mossi et des Sissala sur les espèces agroforestières, qui se distinguent nettement de celles des Dagara. Pourtant, la cohabitation depuis un siècle entre les deux dernières ethnies (Savonnet, 1970) aurait pu générer une diffusion de part et d'autre des connaissances sur les plantes. Les Mossi sont toutefois des migrants plutôt récents, qui se sont massivement installés dans le bassin versant suite aux sécheresses des années 1980, et à la faveur de l'opération « retour au pays natal » faisant suite à la crise ivoirienne des années 2000. Ils n’ont pas de maîtrise foncière et adoptent les pratiques de conservation des Sissala, chez lesquels ils obtiennent les droits d'usage sur la terre. En outre le faible nombre d'espèces ligneuses associées aux cultures chez les Dagara peut être lié au fait qu'ils ont recours aux bois des arbres présents dans les champs pour la préparation quotidienne du dolo, bière de mil qui exige de grandes quantités de bois-énergie.

Des individus de $V$. paradoxa sont présents dans tous les champs et sont les plus gros pourvoyeurs de services d'approvisionnement dans la zone d'étude. Le rôle de cette espèce dans les usages quotidiens des populations en Afrique de l'Ouest est bien documenté (Moore, 2008 ; Orwa et al., 2009 ; Guigma et al., 2012).

Les espèces $B$. aegyptiaca, Pericopsis laxiflora, D. mespiliformis, B. africana, Lophira lanceolata, Daniellia oliveri, Acacia spp. et les Combretaceae sont peu fréquentes dans les exploitations agricoles du bassin versant de Boura. Les individus de ces espèces, pourtant d'intérêt socio-économique, sont parfois

Figure 6.

Indice culturel d'importance des catégories de services

écosystémiques selon les groupes ethniques. 

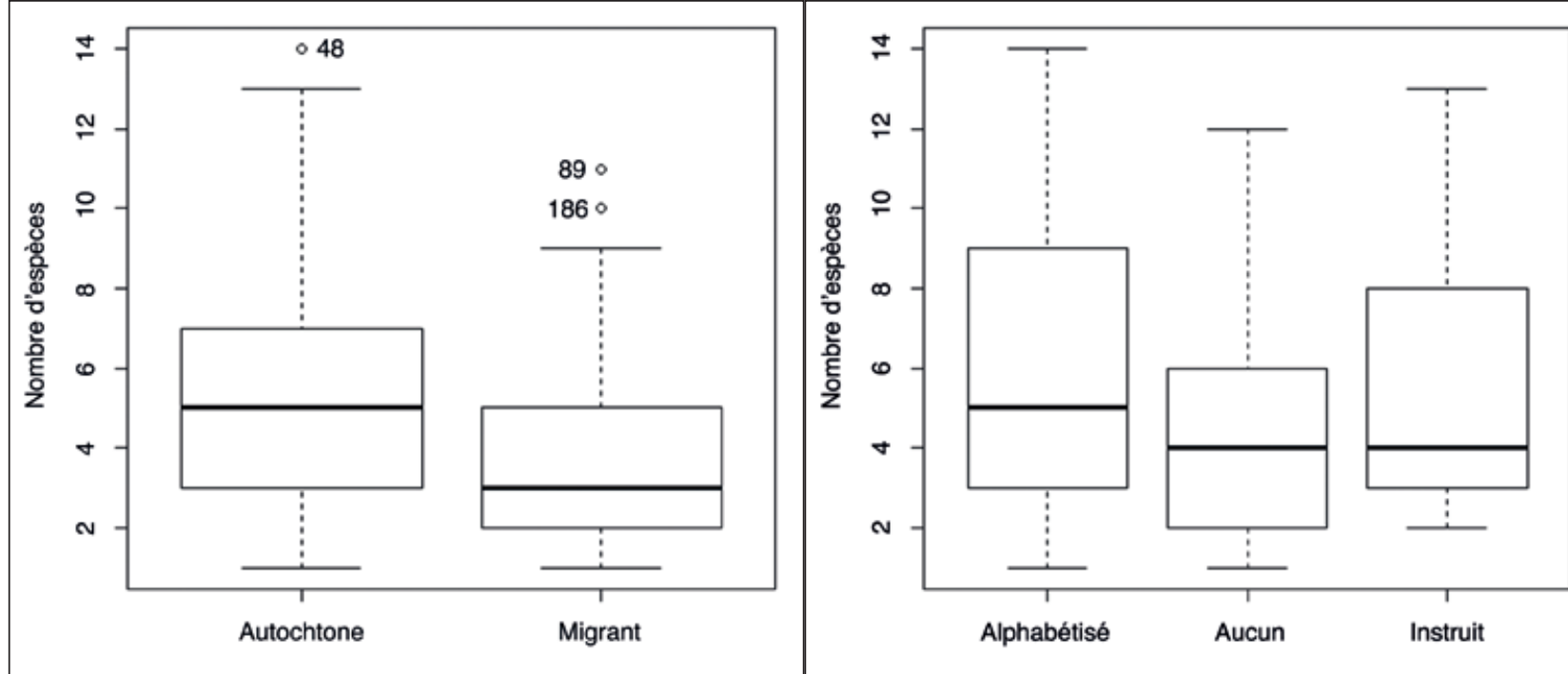

Statut résidentiel $a b$

Niveau d'éducation
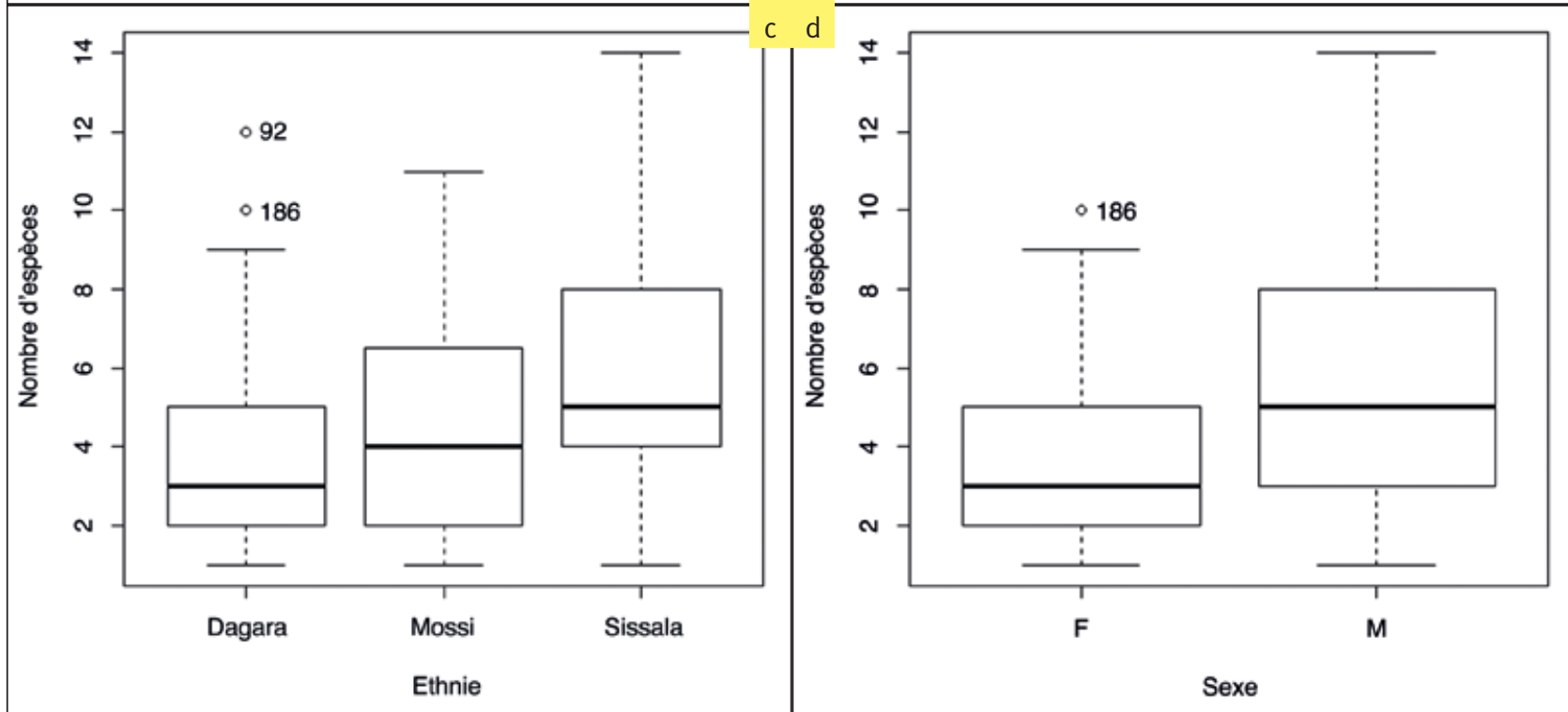

Figure 7.

Répartition du nombre d'espèces agroforestières selon le statut résidentiel (a) et le niveau d'éducation (b), l'ethnie (c), le sexe (d) ( $F$ : féminin ; $M$ : masculin).

Tableau III.

Effets des facteurs socio-économiques sur la perception des espèces agroforestières.

\begin{tabular}{|l|r|r|l|}
\hline Facteur & $\begin{array}{c}\text { Kruskal-Wallis } \\
\mathbf{X}^{\mathbf{2}}\end{array}$ & $\mathbf{d f}$ & $\begin{array}{c}\mathbf{p} \text {-value } \\
\mathbf{0} \mathbf{0 , 0 5}\end{array}$ \\
\hline Classe d'âge & 47,39 & 11 & $1,84 \mathrm{E}-06$ \\
\hline Ethnie & 17,96 & 2 & 0,000 \\
\hline Niveau d'éducation & 8,08 & 2 & 0,018 \\
\hline Sexe & 26,84 & 1 & 0,000 \\
\hline
\end{tabular}

éliminés lors des opérations de préparation des champs. La promotion de $V$. paradoxa (karité) et $P$. biglobosa (néré), abondantes dans la zone, a entraîné une sous-valorisation, voire une négligence à l'égard de certaines espèces ligneuses autochtones.

Dans le même bassin versant, 55 \% des chefs d'exploitation font part de problèmes avec les agents forestiers s'agissant de l'exploitation des arbres. Au Burkina Faso, il n'existe pas de statut spécifique aux arbres des champs, pourtant préservés et protégés pour leurs utilités. Les espèces recensées dans les champs couvrent $60 \%$ c'est à dire $(14 / 23) \star 100$ des espèces intégralement protégées car menacées (MECV, 2004). Cette législation limite ainsi les droits d'usage des paysans pour satisfaire les besoins pour lesquels ces espèces ont été volontairement maintenues dans les champs. 
Tableau IV.

Influence des variables socioéconomiques sur la perception des espèces agroforestières.

\begin{tabular}{l|c|c|c|} 
Variable & $\begin{array}{c}\text { Coefficient } \pm \\
\text { déviation standard }\end{array}$ & z value & $\operatorname{Pr}(>|z|)$ \\
\hline (Intercept) & $0,97 \pm 0,24$ & 4,048 & $5,17 \mathrm{e}-05^{\star \star \star *}$ \\
\hline Âge & $0,00 \pm 0,00$ & 0,784 & 0,4333 \\
\hline Nombre d'actifs & $0,01 \pm 0,01$ & 1,041 & 0,2977 \\
\hline NAE & $0,03 \pm 0,01$ & 2,010 & $0,0444^{\star}$ \\
\hline Taille du ménage & $0,02 \pm 0,01$ & 2,447 & $0,0144^{\star}$ \\
\hline Age x NAE & $0,00 \pm 0,00$ & $-1,685$ & 0,0919
\end{tabular}

Signification des codes : ${ }^{\star \star \star}$ : très significatif ; ${ }^{*}$ : significatif ; $\mathrm{NAE}$ : nombre d'années d'expérience.

Tableau V.

Classification des espèces inventoriées dans les champs.

\begin{tabular}{|c|c|c|c|c|c|c|c|c|}
\hline \multirow{3}{*}{$\begin{array}{l}\text { Espèces } \\
\text { Vitellaria paradoxa C.F. Gaertn. }\end{array}$} & \multirow{3}{*}{$\begin{array}{l}\text { Familles } \\
\text { Sapotaceae }\end{array}$} & \multicolumn{4}{|c|}{ Indice culturel des catégories de services ICs } & \multirow{3}{*}{$\begin{array}{r}\text { ICt } \\
10,45\end{array}$} & \multirow{3}{*}{$\begin{array}{c}\text { Rang } \\
1 \\
1\end{array}$} & \multirow{3}{*}{$\begin{array}{l}\text { Statu } \\
\text { au BF } \\
+ \\
+\end{array}$} \\
\hline & & Provision & Régulation & Support & Culturel & & & \\
\hline & & 4,59 & 3,36 & 1,68 & 0,82 & & & \\
\hline Parkia biglobosa (Jacq.) R. Br. ex G. Don & Fabaceae-Mimosoideae & 3,10 & 2,75 & 1,37 & 0,58 & 7,80 & 2 & + \\
\hline Detarium microcarpum Guill. \& Perr. & Fabaceae-Caesalpinioideae & 0,99 & 0,85 & 0,44 & 0,08 & 2,36 & 3 & 0 \\
\hline Lannea microcarpa Engl. \& K. Krause & Anacardiaceae & 0,88 & 0,88 & 0,43 & 0,17 & 2,36 & 4 & 0 \\
\hline Tamarindus indica $\mathrm{L}$. & Fabaceae-Caesalpinioideae & 0,95 & 0,79 & 0,43 & 0,12 & 2,29 & 5 & + \\
\hline Afzelia africana Sm. ex Pers. & Fabaceae-Caesalpinioideae & 0,79 & 0,76 & 0,36 & 0,21 & 2,13 & 6 & + \\
\hline Saba senegalensis (A. DC.) Pichon & Apocynaceae & 0,62 & 0,57 & 0,29 & 0,06 & 1,54 & 7 & 0 \\
\hline Bombax costatum Pellegr. \& Vuill. & Malvaceae & 0,44 & 0,48 & 0,25 & 0,06 & 1,23 & 8 & + \\
\hline Pterocarpus erinaceus Poir. & Fabaceae-Faboideae & 0,51 & 0,36 & 0,19 & 0,01 & 1,07 & 9 & + \\
\hline Khaya senegalensis (Desr.) A. Juss. & Meliaceae & 0,51 & 0,33 & 0,17 & 0,03 & 1,05 & 10 & + \\
\hline $\begin{array}{l}\text { Diospyros mespiliformis Hochst. } \\
\text { ex A. DC. }\end{array}$ & Ebenaceae & 0,37 & 0,40 & 0,17 & 0,08 & 1,03 & 11 & 0 \\
\hline Strychnos spinosa Lam. & Loganiaceae & 0,36 & 0,39 & 0,19 & 0,02 & 0,96 & 12 & 0 \\
\hline Burkea africana Hook. & Fabaceae-Caesalpinioideae & 0,40 & 0,28 & 0,14 & 0,01 & 0,83 & 13 & 0 \\
\hline Faidherbia albida (Delile) A. Chev. & Fabaceae-Mimosoideae & 0,32 & 0,24 & 0,11 & 0,07 & 0,74 & 14 & + \\
\hline Azadirachta indica A. Juss. & Meliaceae & 0,33 & 0,22 & 0,10 & 0,01 & 0,66 & 15 & 0 \\
\hline Lannea acida A. Rich. & Anacardiaceae & 0,21 & 0,27 & 0,11 & 0,05 & 0,64 & 16 & 0 \\
\hline Ficus sycomorus L. & Moraceae & 0,24 & 0,21 & 0,11 & 0,00 & 0,56 & 17 & 0 \\
\hline Terminalia avicennioides Guill. \& Perr. & Combretaceae & 0,26 & 0,17 & 0,09 & 0,00 & 0,52 & 18 & 0 \\
\hline Cordia myxa L. & Boraginaceae & 0,23 & 0,18 & 0,09 & 0,01 & 0,51 & 19 & 0 \\
\hline Balanites aegyptiaca (L.) Delile & Zygophyllaceae & 0,22 & 0,18 & 0,10 & 0,00 & 0,51 & 20 & 0 \\
\hline Sterculia setigera Delile & Malvaceae & 0,14 & 0,15 & 0,07 & 0,03 & 0,38 & 21 & 0 \\
\hline Daniellia oliveri (Rolfe) Hutch. \& Dalziel & Fabaceae-Caesalpinioideae & 0,15 & 0,14 & 0,07 & 0,01 & 0,36 & 22 & 0 \\
\hline Adansonia digitata $\mathrm{L}$. & Malvaceae & 0,12 & 0,15 & 0,08 & 0,00 & 0,36 & 23 & + \\
\hline $\begin{array}{l}\text { Piliostigma thonningii (Schumach.) } \\
\text { Milne-Redh. }\end{array}$ & Fabaceae-Caesalpinioideae & 0,17 & 0,09 & 0,07 & 0,01 & 0,34 & 24 & 0 \\
\hline Mangifera indica L. & Anacardiaceae & 0,11 & 0,10 & 0,06 & 0,01 & 0,28 & 25 & 0 \\
\hline Pericopsis laxiflora (Benth.) Meeuwen & Fabaceae-Caesalpinioideae & 0,11 & 0,08 & 0,05 & 0,00 & 0,25 & 26 & 0 \\
\hline
\end{tabular}


Tableau V (suite).

Espèces
Indice culturel des catégories de services ICs Provision Régulation Support Culturel
ICt Rang Statut au BF

\begin{tabular}{|c|c|c|c|c|c|c|c|c|}
\hline Gardenia erubescens Stapf \& Hutch. & Rubiacea & 0,08 & 0,09 & 0,04 & 0,01 & 0,23 & 27 & 0 \\
\hline Moringa oleifera Lam. & Moringaceae & 0,09 & 0,08 & 0,05 & 0,00 & 0,21 & 28 & 0 \\
\hline $\begin{array}{l}\text { Pseudocedrela kotschyi (Schweinf.) } \\
\text { Harms }\end{array}$ & Meliaceae & 0,10 & 0,07 & 0,04 & 0,00 & 0,21 & 29 & 0 \\
\hline Mitragyna inermis (Willd.) Kuntze & Rubiaceae & 0,09 & 0,07 & 0,03 & 0,01 & 0,20 & 30 & 0 \\
\hline Acacia dudgeonii Craib ex Holl. & Fabaceae-Mimosoideae & 0,08 & 0,06 & 0,04 & 0,01 & 0,19 & 31 & 0 \\
\hline $\begin{array}{l}\text { Crossopteryx febrifuga (Afzel. } \\
\text { ex G. Don) Benth. }\end{array}$ & Rubiaceae & 0,08 & 0,07 & 0,03 & 0,00 & 0,17 & 32 & 0 \\
\hline Anacardium occidentale L. & Anacardiaceae & 0,06 & 0,07 & 0,04 & 0,00 & 0,16 & 33 & 0 \\
\hline Lophira lanceolata Tiegh. ex Keay & Ochnaceae & 0,07 & 0,05 & 0,03 & 0,00 & 0,15 & 34 & 0 \\
\hline $\begin{array}{l}\text { Anogeissus leiocarpa (DC.) } \\
\text { Guill. \& Perr. }\end{array}$ & Combretaceae & 0,06 & 0,05 & 0,02 & 0,01 & 0,14 & 35 & + \\
\hline Ficus dicranostyla Mildbr. & Moraceae & 0,05 & 0,06 & 0,03 & 0,01 & 0,14 & 36 & 0 \\
\hline $\begin{array}{l}\text { Xeroderris stuhlmannii (Taub.) } \\
\text { Mendonça \& E. C. Sousa }\end{array}$ & Fabaceae-Faboideae & 0,06 & 0,06 & 0,03 & 0,00 & 0,14 & 37 & 0 \\
\hline Erythrina senegalensis DC. & Fabaceae-Faboideae & 0,05 & 0,06 & 0,03 & 0,00 & 0,14 & 38 & 0 \\
\hline Lannea velutina A. Rich. & Anacardiaceae & 0,05 & 0,04 & 0,02 & 0,00 & 0,12 & 39 & 0 \\
\hline Parinari curatellifolia Planch. ex Benth. & Chrysobalanaceae & 0,06 & 0,03 & 0,01 & 0,00 & 0,10 & 40 & 0 \\
\hline Ficus ovata Vahl & Moraceae & 0,03 & 0,04 & 0,02 & 0,01 & 0,10 & 41 & 0 \\
\hline Quassia undulata (Guill. \& Perr.) F. Dietr. & Simaroubaceae & 0,05 & 0,04 & 0,01 & 0,00 & 0,10 & 42 & 0 \\
\hline Securidaca longipedunculata Fres. & Polygalaceae & 0,04 & 0,03 & 0,01 & 0,00 & 0,09 & 43 & 0 \\
\hline Trichilia emetica Vahl & Meliaceae & 0,04 & 0,04 & 0,02 & 0,00 & 0,09 & 44 & 0 \\
\hline Crateva adansonii DC. & Capparaceae & 0,03 & 0,03 & 0,02 & 0,00 & 0,08 & 45 & 0 \\
\hline Vitex doniana Sweet & Lamiaceae & 0,03 & 0,02 & 0,02 & 0,00 & 0,07 & 46 & + \\
\hline Combretum molle R. Br. ex G. Don & Combretaceae & 0,03 & 0,02 & 0,02 & 0,00 & 0,07 & 47 & 0 \\
\hline Cassia siamea Lam. & Fabaceae-Caesalpinioideae & 0,03 & 0,02 & 0,01 & 0,00 & 0,06 & 48 & 0 \\
\hline Isoberlinia doka Craib \& Stapf & Fabaceae-Caesalpinioideae & 0,03 & 0,01 & 0,01 & 0,00 & 0,06 & 49 & 0 \\
\hline Prosopis africana (Guill. \& Perr.) Taub. & Fabaceae-Mimosoideae & 0,03 & 0,02 & 0,01 & 0,00 & 0,06 & 50 & + \\
\hline Ceiba pentandra (L.) Gaertn. & Malvaceae & 0,02 & 0,02 & 0,01 & 0,00 & 0,05 & 51 & + \\
\hline Dichrostachys cinerea (L.) Wight \& Arn. & Fabaceae-Mimosoideae & 0,02 & 0,02 & 0,01 & 0,00 & 0,05 & 52 & 0 \\
\hline Hymenocardia acida Tul. & Phyllanthaceae & 0,02 & 0,02 & 0,01 & 0,00 & 0,05 & 53 & 0 \\
\hline Pteleopsis suberosa Engl. \& Diels & Combretaceae & 0,02 & 0,02 & 0,01 & 0,00 & 0,05 & 54 & 0 \\
\hline Entada africana Guill. \& Perr. & Fabaceae-Mimosoideae & 0,02 & 0,02 & 0,01 & 0,00 & 0,05 & 55 & 0 \\
\hline Ficus platyphylla Del. & Moraceae & 0,02 & 0,01 & 0,01 & 0,00 & 0,05 & 56 & 0 \\
\hline Gmelina arborea Roxb. & Lamiaceae & 0,02 & 0,02 & 0,01 & 0,00 & 0,05 & 57 & 0 \\
\hline Crescentia cujete L. & Bignoniaceae & 0,01 & 0,02 & 0,01 & 0,00 & 0,04 & 58 & 0 \\
\hline Flacourtia indica Willd. & Salicaceae & 0,02 & 0,02 & 0,00 & 0,00 & 0,04 & 59 & 0 \\
\hline Ximenia americana L. americana & Ximeniaceae & 0,02 & 0,01 & 0,01 & 0,00 & 0,04 & 60 & + \\
\hline $\begin{array}{l}\text { Zanthoxylum zanthoxyloides (Lam.) } \\
\text { Watermann }\end{array}$ & Rutaceae & 0,02 & 0,02 & 0,00 & 0,00 & 0,04 & 61 & 0 \\
\hline Tectona grandis L. f. & Lamiaceae & 0,02 & 0,00 & 0,00 & 0,00 & 0,02 & 62 & 0 \\
\hline Psidium guajava L. & Myrtaceae & 0,01 & 0,00 & 0,00 & 0,00 & 0,01 & 63 & 0 \\
\hline Carica papaya L. & Caricaceae & 0,01 & 0,00 & 0,00 & 0,00 & 0,01 & 64 & 0 \\
\hline Total & & 18,71 & 15,70 & 7,90 & 2,57 & 44,88 & & \\
\hline
\end{tabular}

+ : espèce intégralement protégée au Burkina Faso ; 0 : espèce non intégralement protégée. 


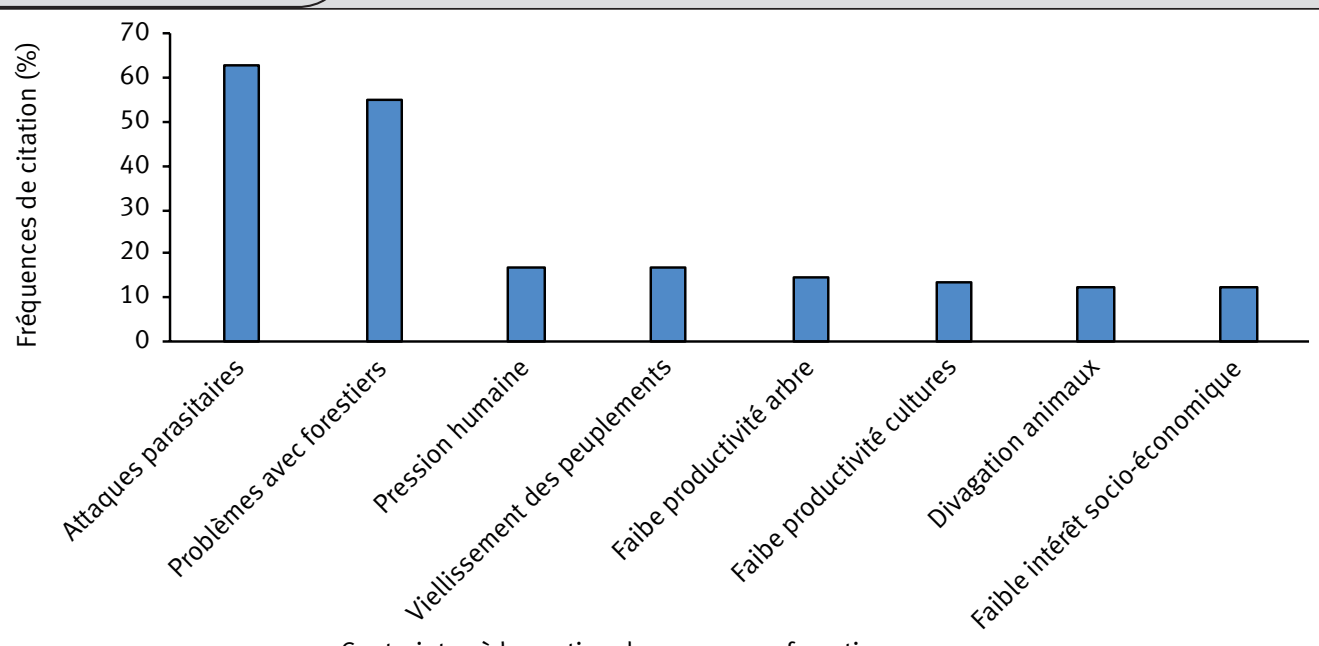

Contraintes à la gestion des parcs agroforestiers

Figure 8.

Contraintes de gestion des parcs agroforestiers.

L'indice culturel d'importance montre que la perception des catégories de services écosystémiques ne varie pas significativement selon les ethnies. Cela suggère que les communautés partagent des connaissances liées aux services des arbres de champs.

Les services d'approvisionnement (ICp $=18,7)$ sont les plus importants parmi les quatre catégories recensées. Ouedraogo et al., (2014) ont rapporté des résultats similaires dans les aires protégées à l'est du Burkina Faso. Ces résultats suggèrent que les ligneux sont maintenus dans les champs pour leur valeur utilitaire (Bounkoungou et al., 1994 ; Boffa, 2000 ; Yaméogo et al., 2005 ; Dao, 2013). Les produits des arbres sont utilisés comme base de nourriture pendant les périodes de soudure (Belem et al., 2008 ; Guigma et al., 2012 ; Thiombiano et al., 2012b), de fourrage (photo 4), de bois énergie et de service, etc. (Bationo et al.,

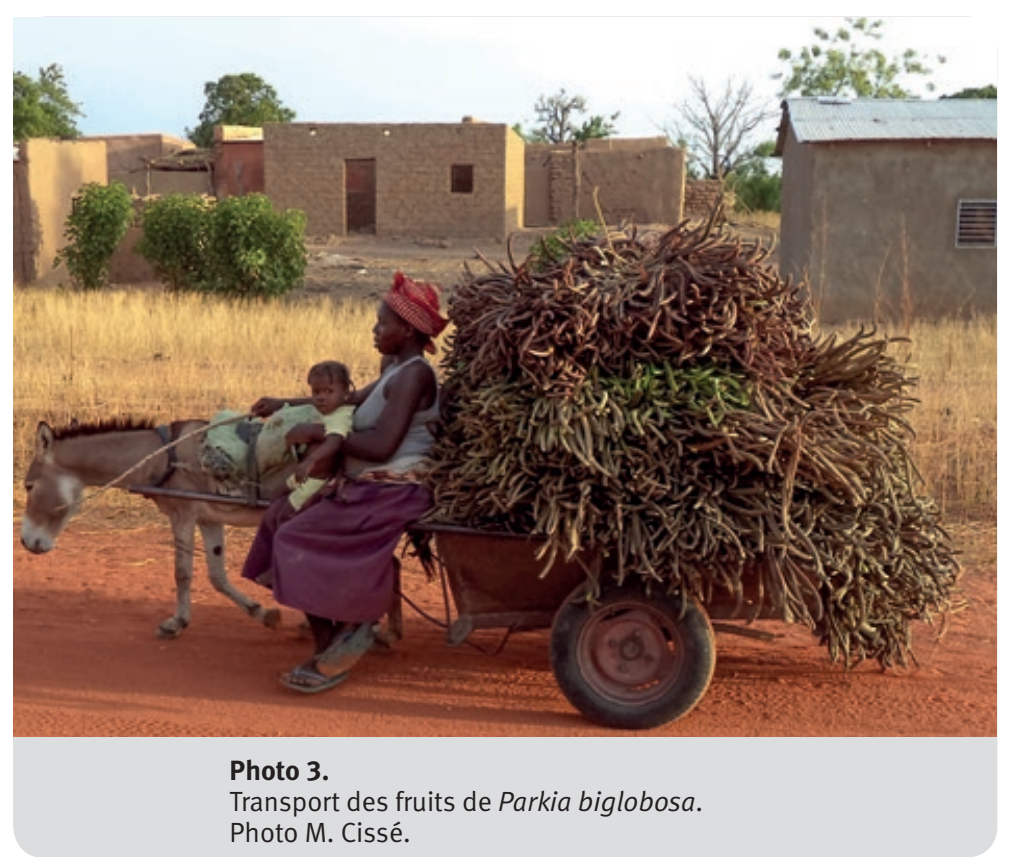

2004 ; Larwanou et al., 2010). Ces produits obtenus des arbres de champs sont aussi commercialisés et procurent des revenus substantiels aux ménages (Boffa, 2000). L'importance alimentaire des espèces agroforestières corrobore les travaux de Raison (1988) qui présentent l'alimentation humaine comme l'une des fonctions principales des parcs agroforestiers en Afrique. Les ligneux agroforestiers spécifiquement épargnés pour leur usage en pharmacopée ont été cités comme espèces rares, menacées de disparition selon $42,5 \%$ des enquêtés. Ces espèces sont Trichilia emetica, Prosopis africana, Xeroderris sthulmanii et Lophira lanceolata. Les espèces pourvoyeuses de fibres sont surtout Saba senegalensis et Pteleopsis suberosa.

L'ombrage (54\%) représente le service le plus important dans les services de support. Au lieu d'être une contrainte pour les cultures associées (Yaméogo et al., 2005), les paysans de la zone d'étude produisent, à l'ombre des arbres, des piments, de l'igname ou des aubergines. Toutes les espèces agroforestières procurent un ombrage, alors que la perception d'autres catégories de services (alimentation, fourrage, pharmacopée) sont spécifiques et dépendent souvent de l'expérience des chefs d'exploitation. En outre, la perception des services de fertilisation serait liée aux rôles d'espèces comme $F$. albida, et à l'utilisation de D. microcarpum comme paillage.

De nombreuses espèces procurent des services culturels. A. africana, L. acida et $S$. setigera sont des espèces à connotation mystique et sont perçues comme abritant des génies. Les individus de ces espèces sont épargnés des coupes pour se préserver d'événements malheureux. Bationo et al. (2010) ont rapporté des comportements similaires à l'égard du baobab (Adansonia digitata) chez des populations du nord du Burkina Faso. Faidherbia albida sert en outre de symbole identitaire chez de nombreux 
Fontès J., Guinko S., 1995. Carte de la végétation et de l'occupation du sol au Burkina Faso. Notice explicative. Ministère de la Coopération française, Projet campus (8813101). Toulouse, France, Université Paul Sabatier, 67 p.

Ganaba S., 2010. Importance de la recherche scientifique dans la conservation. In : Thiombiano A., Kampmann D. (éds). Atlas de la biodiversité de l'Afrique de l'Ouest, tome II, volume II : Burkina Faso. Ouagadougou et Frankfurt/Main, 422-429. https://www. uni-frankfurt.de $/ 47671163 / \mathrm{Cl}$ Atlas complete.pdf

Guigma Y., Zerbo P., Millogo-Rasilodimby J., 2012. Utilisation des espèces spontanées dans trois villages contigus du Burkina Faso. Tropicultura, 30 (4) : 230-235.

Hervouët J.-P., 1993. Faidherbia albida : un témoin des mutations agraires. In : Vandenbeldi R. J., Renard C. (éds). Faidherbia albida dans les zones tropicales semi-arides d'Afrique de l'Ouest. Comptes rendus d'un atelier du 22 au 26 avril 1991, Niamey, Niger, Institut international de recherche sur les cultures des zones tropicales semiarides, Centre international de recherche en agroforesterie, 165-169. http://horizon.documentation.ird.fr/exl-doc/pleins textes/pleins textes 6/b fdi 47-48/010011408.pdf

Houéhanou D. T., Assogbadjo A. E., Chadare F. J., Zanvo S., Sinsin B., 2015. Approches méthodologiques synthétisées des études d'ethnobotanique quantitative en milieu tropical. Annales des Sciences Agronomiques, 19 (spécial Projet Undesert-UE) : 173-193.

Hounsode D. M. T., Assogbadjo A. E., Houéhanou D. T., Glele Kakaï R. L., Agbangla C., 2016. Facteurs socioéconomiques influençant l'usage des raphias au Bénin (Afrique de l'Ouest). Rev. CAMES, 4 (01) : 1-17. http://bj.chm-cbd.net/ressources/suivi-de-la-spanb/ axe-strategique-3.-mise-en-place-d-un-mecanisme-de-planification-de-gestion-des/strategique-e/objectif-14/facteurs-socioeconomiques-influencant-l-usage-des-raphias-au-benin-afrique-de-l Institut national de la statistique et de la démographie (INSD), 2006. Recensement global de la population et de l'habitat. Ouagadougou, Burkina Faso. www.insd.bf

Kalinganire A., Kaya B., Niang A., Kindt R., Muraya P., Coe R., 2005. Caractérisation de la biodiversité ligneuse dans les zones en marge du désert : Manuel de procédures. Nairobi, Kenya, ICRAF, Occasional Paper 3, $38 \mathrm{p}$.

Kristensen M., Lykke A. M., 2003. Informant-based valuation of use and conservation preferences of savanna trees in Burkina Faso. Economic Botany, 57 (2): 203-217.

Larwanou M., Oumarou I., Snook L., Danguimbo I., Eyog-Matig O., 2010. Pratiques sylvicoles et culturales dans les parcs agroforestiers suivant un gradient pluviométrique nord-sud dans la région de Maradi au Niger. Tropicultura, 28 (2) : 115-122. http://www.forestday.org/fileadmin/downloads/fd5/Tropicultura.pdf

Millenium Ecosystem Assessment (MEA), 2005. Ecosystems and human well-being: Scenarios. Washington DC, USA, Island Press, $596 \mathrm{p}$.

Ministère de l'Environnement et du Cadre de Vie (MECV), 2004. Arrêté $n^{\circ}$ 2004-019 portant détermination de la liste des espèces forestières bénéficiant de mesures de protection particulière. Ouagadougou, Burkina Faso, MECV, 3 p.

Moore S., 2008. The role of Vitellaria Paradoxa in poverty reduction and food security in the Upper East region of Ghana. Earth \& Environment, 3: 209-245.

Ngom D., Charahabil M. M., Sarr O., Bakhoum A., Akpo L. E., 2014. Perceptions communautaires sur les services écosystémiques d'approvisionnement fournis par le peuplement ligneux de la Réserve de Biosphère du Ferlo (Sénégal). Vertigo, 14 (2). https://vertigo. revues.org/15017
Orwa C., Mutua A., Kindt R., Jamnadass R., Simons A., 2009. Agroforestree Database: a tree reference and selection guide version 4.0. World Agroforestry Centre, Kenya. http://www.worldagroforestry. org/af/treedb/

Ouachinou J. M.-A. S., Adomou A. C., Dassou G. H., Yedomonhan H., Tossou G. M., Akoegninou A., 2017. Connaissances et pratiques ethnobotaniques en médecines traditionnelles vétérinaire et humaine au Bénin : similarité ou dissemblance. Journal of Applied Biosciences, 113 : 11174-11183. http://dx.doi.org/10.4314/jab. v113i1.6

Ouedraogo I., Nacoulma B. M. I., Hahn K., Thiombiano A., 2014. Assessing ecosystem services based on indigenous knowledge in south-eastern Burkina Faso (West Africa). International Journal of Biodiversity Science, Ecosystem Services \& Management, 10 p.

Programme national de gestion des terroirs (PNGT 2), 2007. Plan Communal de développement de Boura. PNGT 2, Bénin, 99 p.

Raison J.-P., 1988. Les « parcs » en Afrique : État des connaissances et perspectives de recherche. Paris, France, EHESS, Encyclopédie des techniques agricoles en Afrique tropicale, $117 \mathrm{p}$.

Savonnet G., 1970. Atlas des structures agraires au Sud du Sahara. Pina (Haute-Volta), Paris, Orstom, 4, 66 p. + cartes et photos.

Savadogo S., Sop T. K., Thiombiano A., 2017. Sacred and totemic plants among thirty two ethnic groups in Burkina Faso: implications for biodiversity conservation. Annales des Sciences Agronomiques, 21 (1): 89-120. https://www.ajol.info/index.php/asab/article/ view/151395

Tardío J., Pardo-de-Santayana M., 2008. Cultural Importance Indices: A Comparative Analysis Based on the Useful Wild Plants of Southern Cantabria (Northern Spain). Economic Botany, 62 (1): 24-39.

Thiombiano A., Schmidt M., Dressler S., Ouedraogo A., Hahn K., Zizka G., 2012a. Catalogue des plantes vasculaires du Burkina Faso. Boissiera, $65: 1-391$.

Thiombiano D. N. E., Lamien N., Dibong D. S., Boussim I. J., Belem B., 2012b. Le rôle des espèces ligneuses dans la gestion de la soudure alimentaire au Burkina Faso. Sécheresse, 23 : 86-93.

Traoré L., Ouedraogo I., Ouedraogo A., Thiombiano A., 2011. Perceptions, usages et vulnérabilité des ressources végétales ligneuses dans le Sud-Ouest du Burkina Faso. International Journal of Biological and Chemical Sciences, 5 (1) : 258-278.

Yaméogo G., Yélémou B., Traoré D., 2005. Pratique et perception paysannes dans la création de parc agroforestier dans le terroir de Vipalogo (Burkina Faso). Biotechnologie, Agronomie, Société et Environnement, 9 (4) : 241-248. http://www.pressesagro.be/base/ text/v9n4/241.pdf

Zomboudre G., Zombre G., Ouedraogo M., Guinko S., Roymacauley H., 2005. Productivité du maïs (Zea mays L.) associé au karité (Vitellaria paradoxa Gaertn.) dans un système agroforestier. Rev. CAMES, Série A, $3: 19-27$.

Bois et Forêts des Tropiques - Revue scientifique du Cirad
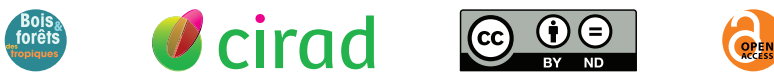

Cirad - Campus international de Baillarguet, 34398 Montpellier Cedex 5, France - Contact : bft@cirad.fr - ISSN : L-0006-579X 